\title{
Mammalian cell viability on hydrophobic and superhydrophobic fabrics
}

\author{
M. Carmen Morán ${ }^{\mathrm{a}, \mathrm{b}},{ }_{*}$, Guillem Ruano $^{\mathrm{a}}$, Francesca Cirisano ${ }^{\mathrm{c}}$, Michele Ferrari ${ }^{\mathrm{c}, * *}$ \\ ${ }^{a}$ Departament de Bioquímica i Fisiologia, Secció de Fisiologia - Facultat de Farmàcia i Ciències de l'Alimentació, Universitat de Barcelona, Avda. Joan XXIII s/n, 08028 Barcelona, Spain \\ ${ }^{\mathrm{b}}$ Institut de Nanociència i Nanotecnologia - IN ${ }^{2} U B$, Universitat de Barcelona, Avda. Joan XXIII $s / n, 08028$ Barcelona, Spain \\ ${ }^{c}$ CNR-ICMATE Istituto di Chimica della Materia Condensata e di Tecnologie per l'Energia, via De Marini, 6, 16149 Genova, Italy
}

\section{ART ICLE INFO}

\section{Keywords:}

Hydrophobic

Superhydrophobic

Coating

Fabric

In vitro cell viability

\begin{abstract}
A B S T R A C T
Surface properties like hydrophobicity and morphology of the substrate are essential for cell proliferation affecting its growth, survival and also for its communication with other cells on fabrics. The combination of low surface energy and a specific surface morphology (micro/nano-roughness) leads to significantly less wettable surfaces, known as superhydrophobic characterized by high contact angle above $150^{\circ}$ and a very small hysteresis. Such high water repellent coatings feature small area available to be exploited in many applications where interactions with aqueous environment are strongly to be avoided. In this work, the authors have investigated the influence of coating polyester fabric at different degree of hydrophobicity by mixed organic-inorganic coating with moderated to highly water repellence. Depending on the coating composition and structure, the hydrophobicity of the fabric can be finely modulated by an easy-to-prepare method applicable to commercial, low cost fabric substrates providing advanced performance. In vitro experiments have been performed in order to establish the influence of surface modification on adhesion of representative model mammalian cell lines such as 3T3 fibroblasts, HaCaT keratinocytes and HeLa epithelial carcinoma cells. The obtained results suggested that, in addition to the chemistry and morphology of the coating, the characteristics of the substrate are important parameters on the final cell viabilities.
\end{abstract}

\section{Introduction}

Interfacial properties play a vital role in biology and medicine with most biological reactions occurring at surfaces and interfaces [1]. Consequently, cell adhesion and spreading control are fundamental and essential requirements for biomaterials frequently used in biomedical devices [2]. Improving biocompatibility and functionality is often accomplished by surface modification of materials with the aim to maintain their properties in terms of stability, wear resistance and a low friction for specific applications [3].

Culturing cells out of their natural niches requires a comprehensive insight into the biochemical and biophysical rules that dictate cell biology. The majority of the cells derived from vertebrates, with the exception of hematopoietic cell lines and a few others, are anchorage-dependent and have to be cultured on a suitable substrate that is specifically treated to allow cell adhesion and spreading. The use of polystyrene which has been chemically modified to enhance cell attachment, i.e.
Tissue-Culture Polystyrene (TCPS) plates is a widely accepted practice for the cell culture of most mammalian cells.

Cell attachment can be considered as a result of a complex process, influenced by several factors coming from cell behavior, material surface properties, adsorption of surface active material present in the extracellular matrix (ECM) and environmental conditions. When a fabric undergoes to a surface modification, coating material has to be studied as a function of its surface properties like hydrophobicity, charge, roughness, softness and chemical composition of the biomaterial itself [4]. Concerning surface hydrophobicity, it is well known that this parameter is a key factor to govern cell response and that cell adhesion on the surface is favored in the more hydrophilic surface of material films $[5,6]$. The material surface topography is another important factor influencing cell adhesion and behavior. Indeed, roughness modulates the biological response of tissues in contact with the substrate. Literature papers report that cells grown on microrough surfaces were stimulated towards differentiation; as shown by their gene expression in comparison with cells growing on smooth surfaces and, nevertheless, the re-

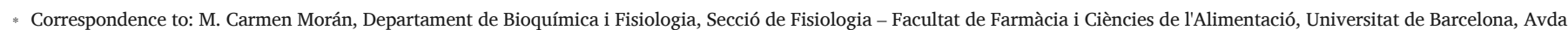
Joan XXIII s/n, 08028 Barcelona, Spain.

** Corresponding author.

Email addresses: mcmoranb@ub.edu (M.Carmen Morán); michele.ferrari@ge.icmate.cnr.it (M. Ferrari)
} 
sponse of cells to roughness is different depending on the cell size and type [4]. The selectivity of cells for surface roughness could be highly advantageous on the development of implanted devices. Moreover, cell attachment, proliferation and differentiation could be modulated by the substrate rigidity to a degree dependent upon the substrate stiffness in relation to the stiffness of the native tissue $[7,8]$.

Highly water repellent coatings with wettability properties known as superhydrophobicity (SH) are related to surfaces with contact angle above $150^{\circ}$ and a very small hysteresis. The small area available for these surfaces when in contact with water addresses to be exploited in many applications where interactions with aqueous environment are usually strongly to be avoided. The combination of low surface energy and the existence of a specific surface morphology (micro/nano-roughness) coexist, lead to significantly less wettable surfaces [9-12].

There is significant interest in the design and development of superhydrophobic materials for biomedical applications, including cell scaffolds, non-fouling surfaces to prevent binding of protein, cells, and/ or bacteria, medical diagnostics, and drug delivery, among others [13] where such highly hydrophobic surface interact with tissues, cells, biological fluid and biological molecules. Applications of functionalization of fibers and fabrics by superhydrophobic systems have been recognized for use in clinical treatments and translational medicine. Despite different techniques like electrospinning, sol-gel, plasma polymerization, and coating [14-17] have been employed to produce highly hydrophobic fibers, the surface roughness control and manipulation is still an open topic in textile fields, where, in facts, the biomimetic approach towards self-cleaning properties is preferred after already few decades of research activity on superhydrophobic states [18-20]. Nevertheless, scarce literature data are available on the topic of cell viability in presence of superhydrophobic polyester fabrics comparing preparation method and cell lines and, in most cases, methodologies includes more complex preparation steps [21,22].

When comparing superhydrophobic surfaces with simple hydrophobic and smoother ones, the cells behavior on such surfaces is still to be better defined, because indeed studies can be found reporting discrepancies between enhanced [23-26] and inhibited cell performances $[23,27-29]$. Cell type appears to be a key factor in influencing cell adhesion and spreading [30-34] together with material surface properties namely considering the influence of chemistry and surface topography. Moreover the combination of low surface energy and a specific surface morphology (micro/nano-roughness) lead to significantly less wettable surfaces allowing to control biological substrates adhesion [9-12].

The adhesion of cells to a surface triggers a signaling cascade subsequently regulating diverse cell functions, e.g. viability, proliferation and activation of structural and signaling proteins [35]. The knowledge about the complexity of cell physiology in dependence of the characteristics of biomaterials is of elementary clinical relevance regarding the development of optimal wound dressings [4]. This study aims to the investigation of the effect of surface topography and chemistry (hydrophobic and superhydrophobic surfaces) and substrate dependence (fabric surfaces in comparison with TCPS as reference) on cell behavior of different cell line types (fibroblasts, keratinocytes and epithelial cell lines). An easy-to-prepare method applicable to commercial, low cost fabric substrates has been used. In vitro experiments have been performed in order to establish the influence of chemical and topographical characteristics of polyester fabrics coated by mixed organic-inorganic system resulting in moderated to highly water repellence on cell adhesion and viability of representative model mammalian cell lines such as 3T3 fibroblasts, HaCaT keratinocytes and HeLa epithelial carcinoma cells.

\section{Materials and methods}

\subsection{Materials}

Polyester (PES) textile substrates were kindly provided by wfk Testgewebe-Test Materials and Concepts (Brüggen-Bracht, Germany). Commercially available fluoropolymer was used as received. Fumed silica was purchased from Degussa (Hannover, Germany) with primary particles about $5-30 \mathrm{~nm}$ in size.

2,5-Diphenyl-3,-(4,5-dimethyl-2-thiazolyl) tetrazolium bromide (MTT), neutral red (NRU) dye, and dimethylsulfoxide (DMSO) were obtained from Sigma-Aldrich (St. Louis, MO, USA). Dulbecco's Modified Eagle's Medium (DMEM), fetal bovine serum (FBS), phosphate buffered saline (PBS), L-glutamine solution (200 mM), trypsin-EDTA solution (170,000 U/L trypsin and 0.2 g/L EDTA), and penicillin-streptomycin solution $(10,000 \mathrm{U} / \mathrm{mL}$ penicillin and $10 \mathrm{mg} / \mathrm{mL}$ streptomycin) were purchased from Lonza (Verviers, Belgium). The $75 \mathrm{~cm}^{2}$ flasks, and 24-well cell culture plates were obtained from TPP (Trasadingen, Switzerland). All other reagents were of analytical grade.

\subsection{Methods}

\subsubsection{Surface preparation and characterization}

In this work PES fabric and TCPS tissue culture polystyrene (TCPS) plates have been used after coating procedure to obtain hydrophobic and superhydrophobic properties. PES was the tested base fabric, TCPS was the reference surface and both were coated with a fluoropolymer blend only and with a mixed organic-inorganic dispersion.

PES fabrics of $1 \mathrm{~cm}$ of diameter were modified in order to produce hydrophobic (HS) and superhydrophobic (SHS) surfaces. Dip coating technique with fluoropolymer blend only was used to produce hydrophobic (HS) surfaces whereas fluoropolymer blend in combination with fumed silica nanoparticles was sprayed for superhydrophobic (SHS) surfaces. The latter method was assessed for higher homogeneity and reproducibility of the organic-inorganic dispersion. The dispersion was prepared according to the method described elsewhere [36].

Once dried, surface wettability was investigated by contact angle (CA) by drop shape method by ASTRAview tensiometer [37] allowing real time drop volume control up to tens of $\mu$ l for hysteresis studies and up to 15 frames/s of frame grabbing. Drops of about $5 \mu$ l were deposited on coated and uncoated samples and contact angle was measured up to spreading equilibrium. For SHS drops typically roll off the surface and then CA was measured with the drop still hanging from the capillary tip. CA hysteresis was $<5^{\circ}$ for SHS, higher than $10^{\circ}$ for hydrophobic coatings, while for uncoated fabric samples complete wettability was observed.

SEM observations included morphology studies for both coated and uncoated fabric samples with energy-dispersive X-ray spectroscopy (EDS) elemental maps for following material distribution (F, Si) along the fibers and its homogeneity in the area under investigation.

\subsubsection{Cell cultures}

The murine Swiss albino fibroblast (3T3), the spontaneously immortalized human keratinocyte (HaCaT) and the human epithelial carcinoma (HeLa) cell lines were grown in DMEM medium $(4.5 \mathrm{~g} / \mathrm{L}$ glucose) supplemented with $10 \%(\mathrm{v} / \mathrm{v})$ FBS, $2 \mathrm{mM} \mathrm{L-glutamine,} 100 \mathrm{U} / \mathrm{mL}$ penicillin and $100 \mu \mathrm{g} / \mathrm{mL}$ streptomycin at $37^{\circ} \mathrm{C}, 5 \% \mathrm{CO}_{2}$. Cells were routinely cultured in $75 \mathrm{~cm}^{2}$ culture flasks and were trypsinised using trypsin-EDTA when the cells reached approximately $80 \%$ confluence.

\subsubsection{Cell interactions with surfaces}

The effect of coating on cell viability was evaluated on both solid surfaces and textile fabrics. Thus, conventional 24-well tissue culture 
polystyrene (TCPS) plates were coated by the coating methodology describe before. Concerning the textile fabrics, PES fabrics of $1 \mathrm{~cm}$ of diameter were sterilized in humid vapor $\left(121^{\circ} \mathrm{C}, 1 \mathrm{~atm}\right)$ dried at $60^{\circ} \mathrm{C}$, and properly prepared according to the above described coating methodology. Then, were placed in individual wells of 24-well TCPS plates.

Uncoated TCPS plates (in the absence or presence of any type of textile substrates) and coated TCPS plates were pre-treated before any cell involving assays were conducted. The pre-treatment consisted in the sterilization by UV procedure during $45 \mathrm{~min}$, based on the protocol described by Sharma [38].

The 3T3 $\left(1 \times 10^{5} \mathrm{cell} / \mathrm{mL}\right)$, HaCaT $\left(1 \times 10^{5} \mathrm{cell} / \mathrm{mL}\right)$ and HeLa $\left(5 \times 10^{4} \mathrm{cell} / \mathrm{mL}\right)$ cells were seeded into the coated 24 -well cell culture plates in the absence or presence of fabrics. In order to ensure that cell attachment was not decreased due to medium culture repellence and sample floating, the PES samples were fixed onto the bottom of the culture wells. Cells on uncoated textile substrates constitute cells control in textile substrates. After incubation for $24 \mathrm{~h}$ under $5 \% \mathrm{CO}_{2}$ at $37^{\circ} \mathrm{C}$, the spent medium was replaced with $500 \mu \mathrm{L}$ of fresh medium supplemented with $5 \%$ FBS. After subsequent $24 \mathrm{~h}$, cell viability was determined by the MTT assay. In this assay, living cells reduce the yellow tetrazolium salt MTT to insoluble purple formazan crystals, in a protocol based on that described first by Mossman [39,40]. Thus, media was removed and $500 \mu \mathrm{L}$ of MTT in PBS $(5 \mathrm{mg} / \mathrm{mL})$ diluted 1:10 in medium without FBS and phenol red was then added to the cells. The plates were incubated for a further $3 \mathrm{~h}$, after which the medium was removed. Thereafter, $500 \mu \mathrm{L}$ of DMSO was added to each well to dissolve the purple formazan product. Plates were then placed in a micro titre-plate shaker for $10 \mathrm{~min}$ at room temperature and the absorbance of the resulting solutions was recover in a 96-plate cell culture plate and measured at $550 \mathrm{~nm}$ using a Bio-Rad 550 microplate reader. The effect of each surface on viability was calculated as the percentage of tetrazolium salt reduction by viable cells against the untreated cell control (cells in uncoated TCPS plates).

Changes on cell viability by coating either TCPS or PES substrates were evaluated by considering the ratio between cell viability under both conditions:

Substrate effect $(\mathrm{SE})=\frac{\text { cell viability }(\mathrm{TCPS})}{\text { cell viability }(\mathrm{PES})}$

For comparative purposes, the ratio between cell viability values under uncoated conditions was also determined.
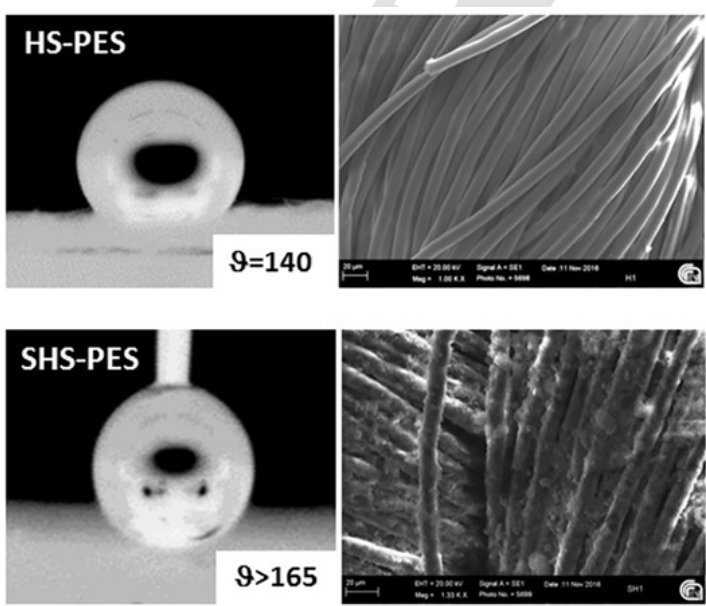

A

\subsubsection{Statistical analyses}

Experiments were performed at least three times on independent occasions unless otherwise stated. Results are expressed as means \pm standard deviation of the mean (SEM). Data were analysed by PASW Statistics 18 software using one-way analysis of variance (ANOVA) with Scheffé post-hoc tests for multiple comparisons. Differences were considered statistically significant at $p<0.05$ or $p<0.005$. In the figures significant differences were illustrated with asterisk (unless otherwise mentioned).

\section{Results and discussion}

\subsection{Physicochemical characterization of the coated surfaces}

The homogeneous distribution of the coating onto yarns and fibers of PES has been evidenced by wettability studies and by EDS maps. In the first case, although the formation of aggregates was evidenced by SEM (Fig. 1C), this didn't result in wettability change or high hysteresis, being CA over $160^{\circ}$ with drops rolling off the surface despite the high heterogeneity of underlying fabric with yarns, holes, macroscopic peak-valley morphology (Fig. 1A). For this reason 3D confocal interferometric profilometry was not performed due to the highly irregular shape of the yarns. EDS maps have been a useful tool to track the single element in the dispersion composition to provide the efficiency of the application method and, in case, to refine or correct potential defect sources. In this case the yarns appear to be homogeneously covered by the polymer alone (HS treatment) and by the organic-inorganic dispersion (SHS treatment) (Fig. 1C).

In case of HS upon application of the polymer alone, the micron and submicron structure of the fabric contributes to a comparatively high CA also considering the polymer surface energy HS $20 \mathrm{mN} / \mathrm{m}$.

In order to work in presence of a substrate whose morphology allows both the coatings to be checked as a reference, TCPS plates were coated with both the coatings. In the case of HS treatment the polymer deposition provides a regular homogeneous surfaces with no defects or asperities with only hydrophobic effect (Fig. 2A). Instead, the morphology of the SHS treatment investigated by SEM and 3D Interferometer confocal profilometry (Fig. 2B and C, respectively), evidences a more homogeneous structure obtained by coating deposition on the as received cell culture plate. The SHS treatment derived from the organic-inorganic coating features a nanometric dual scaled roughness with an average of tens of $\mathrm{nm}$.
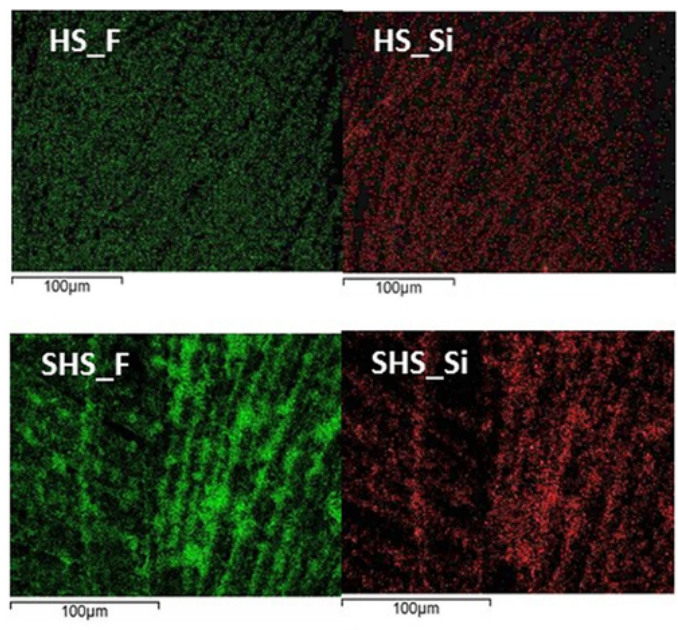

C

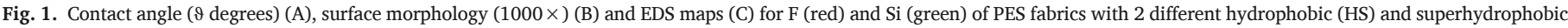
(SHS) treatments. (For interpretation of the references to colour in this figure legend, the reader is referred to the web version of this article.) 


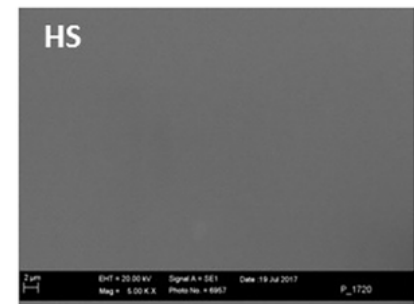

A

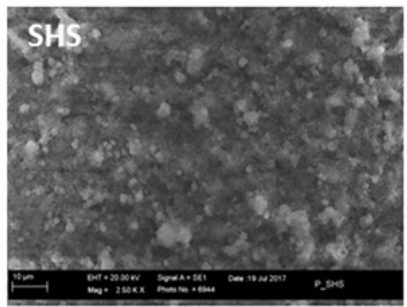

B

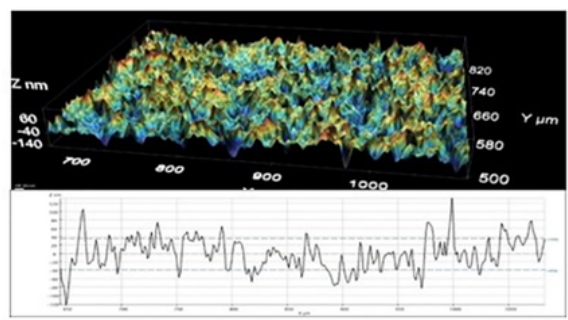

C

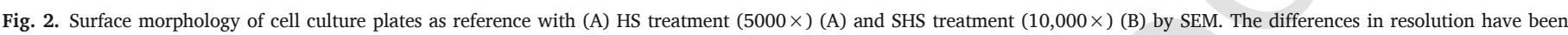
applied to optimize the details available. 3D profilometry of SHS treatment (C).

\subsection{Cell interactions with surfaces}

One of the most common non-epithelial cell line used in short- and long-term toxicological in vitro studies on cytotoxicity, biocompatibility, or mechanisms of cellular uptake of nanoparticles contains 3T3 fibroblasts. These are readily available, undergo contact inhibition, and are closely representative of a physiologic model cell line [41]. On the other hand, HeLa cell line is the oldest and most commonly used human cell line, derived from cervical cancer cells. Since they were put into mass production, HeLa cells have been used for research into cancer, AIDS, effects of radiation and toxic substances, gene mapping, and countless other scientific pursuits $[42,43]$.

The skin is a continuously self-renewing organ that dynamically manages the outside-inside-outside relationships of the human body and actively participates in the host defences. Keratinocytes represent 95\% of the epidermal cells. Primarily, they play the structural and barrier function of the epidermis, but their role in the initiation and perpetuation of skin inflammatory and immunological responses, and wound repair, is also well recognized. The spontaneously immortalized human keratinocytes cell line $\mathrm{HaCaT}$ from adult skin has been proposed as a model for the study of keratinocytes functions. HaCaT is a nontumorigenic monoclonal cell line, adapted to long-term growth without feed-layer or supplemented growth factors. It exhibits normal morphogenesis and expresses all the major surface markers and functional activities of isolated keratinocytes [44,45].

Due to the functional role of fibroblasts, keratinocytes and cancer cells, the interaction of the selected cell lines with the proposed surfaces have been projected. Cell viability on the proposed surfaces was evaluated using a modified in vitro cytotoxicity assay. Due to the adherent character of the selected cell lines, the incubation of first $24 \mathrm{~h}$ constitutes the time necessary for cell adhesion to occur on conventional

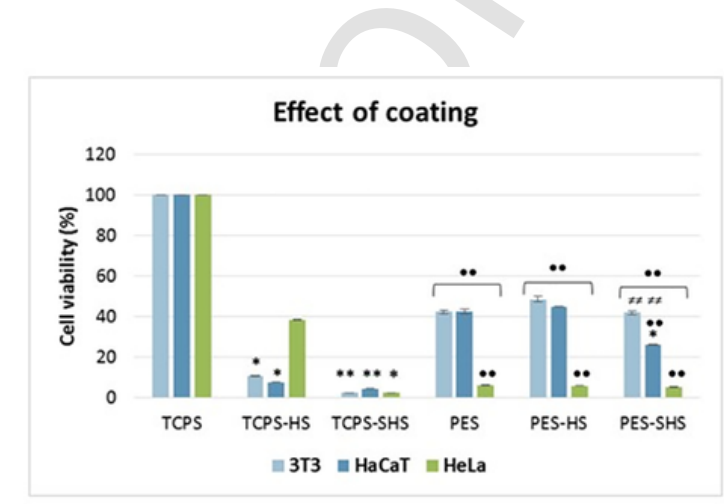

A
TCPS surfaces. Then, the spent medium (with the unattached cells) was replaced with fresh medium and cells were allowed to attach for subsequent $24 \mathrm{~h}$. Cell viability using the MTT assay, as a measurement of cell metabolic activity within the mitochondrial compartment, was used as a measure of cell attachment and growth on the assayed conditions.

Cell viability of 3T3, HaCaT and HeLa cells grown on hydrophobic (HS) and superhydrophobic (SHS) coated TCPS plates for $48 \mathrm{~h}$ was significantly reduced when compared to the control TCPS surface (Fig. 3A). Data are represented as percent of survival cells seeded and grown onto TCPS culture plates that was set at $100 \%$. Cell viabilities of 7.5 (HaCaT), 10.5 (3T3) and 38\% (HeLa) and 2.2-2.4\% (3T3, HeLa) and 4.5\% (HaCaT) were obtained for cells grown in HS and SHS, respectively. In the case of 3T3 and HaCaT cells, significant differences against the uncoated TCPS plates for HS $(p<0.005)$ and SHS $(p<0.001)$ were found. For the tumoral cell line, significant differences $(p<0.005)$ were only found for the HSH treatment. No significant differences between HS and SHS treatments for the same cell line, or differences between cell lines for the same type coating were found.

On growing cells on either uncoated or coated PES fabrics, cell viability was significantly reduced when compared to the control TCPS surfaces (Fig. 3A). In the case of uncoated PES fabric, relative cell viability values ranged between 6.0 and $42 \%$, as a function of cell line. Significant differences between HeLa with any of the non-tumor cell line were found $(p<0.001)$. By modification on the PES fabric by either HS or SHS coating, cell viability responses seem to be dependent on the cell line type. The modification of PES surfaces by HS treatment conferred changes in cell viability ranged between 6 and 48\%, following a similar trend of that observed in the uncoated PES. Interestingly, the modification with SHS treatment induced significant differences among the three cell lines, with viabilities of 42\% (3T3), 26\% (HaCaT) and 5\%(HeLa). In addition, for both non-tumor cells, significant differences between coating type were found $(p<0.001)$.

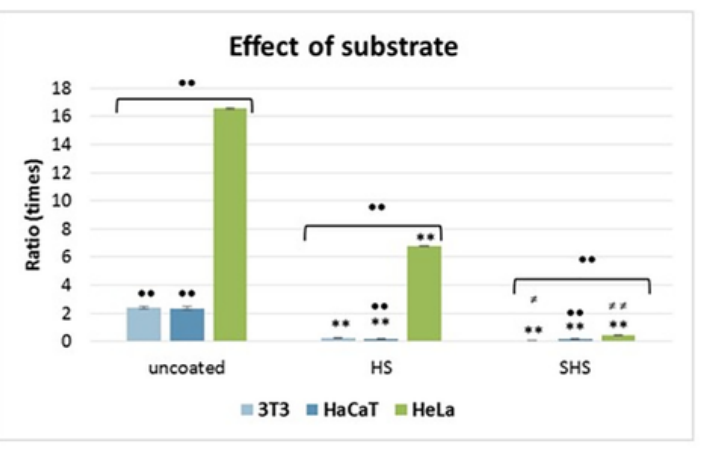

B

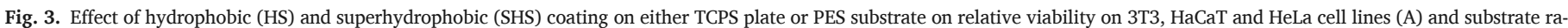

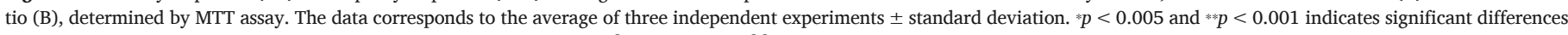

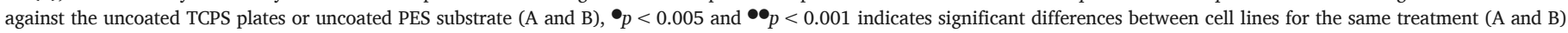
and ${ }^{\neq \neq} p<0.001$ indicates significant differences between the coating type (B). 
The combined effect of surface morphology and chemistry is evidenced in Fig. 3A, where plain TCPS was used as received in comparison with the same surface treated by HS and SHS. While the homogeneity and the hydrophilic nature of the substrate allow the growth of all the three types of cell lines, when treated by SHS the substrate homogeneity allows a coating of nanometric scale roughness to be applied, definitely repelling the aqueous cell culture medium and resulting in an almost complete growth inhibition for all the cell types without differentiation. The difference between the two hydrophobic states is also confirmed in Fig. 3A by the HS treatment where the overall viability in HeLa cells is clearly enhanced with respect to the other cell lines.

On the other side, when the coating is applied on a more rough substrate like the PES fabric with fibers and yarns of few micron size, we observe wettability properties comparable with the more ideal substrate TCPS. Thus, uncoated PES absorbs water featuring a complete wettability while coated PES (both HS and SHS) highly repels it. Nevertheless, the microscopic grooves created within the yarns allow the cell to get into the sites of similar size by sedimentation $[46,47]$ and the preparation of the cell monolayer can take place with a comparable viability with respect to the not treated PES, enhancing few differences for the different cell lines. Despite the role of adhesive proteins is still to be ascertained in details, the monolayer formation appears to be ruled by the substrate morphology, which drives the cell attachment in a mechanism quite independently on fabric coating features in Fig. 3A. We observe a strong viability inhibition for HeLa for all the conditions, while 3T3 and $\mathrm{HaCaT}$ cells decreases slowly or moderately their viability, respectively, according with the change in hydrophobicity.

Changes on 3T3 cell viability when compared to the control TCPS appears to be independent of the coating modification on PES fabric. Reports on the literature have demonstrated that fibroblasts and fibroblastic cells showed a higher strength to resist and proliferate in not so favorable conditions of culture and that is one of the main reasons why it is currently used for standard assays of cytotoxicity [34]. The difference in viability between 3T3 and HaCaT cells in the presence of the hydrophobic fluoropolymers blend could not be related to differences on the surface charge of the cell membrane which is certainly more negative in the case of fibroblasts than in the case of keratinocytes [48]. In addition, recent studies have demonstrated that the negative surface charges are a unique pattern of cancer cells. The negative surface charges were found to be generated from the large quantity of lactate secretion, a known property of all metabolically active cancer cells [49]. However, results found in the present work with HeLa cell line seems to be independent of the surface charge modification of the proposed coated surfaces.

Studies about chemical modification of polystyrene surfaces by UV/ O3 (UVO) irradiation, getting smooth and rough surfaces, have demonstrated that the attachment and proliferation on them depends on cell line type [50]. Compared to standard TCPS, the human primary osteosarcoma cell line (SaOs-2) and the mouse chondrocyte teratocarcinoma-derived cell line (ATDC5) could not proliferate on such surfaces, whereas the mouse lung fibroblastic cell line (L929) exhibited a high proliferation. It has been described that cells of fibroblastic nature as L929 have a higher strength to resist and proliferate in not so favorable conditions of culture [34]. These results are on the light of the obtained results with 3T3 on uncoated and coated PES substrates (Fig. 3A).

The present work demonstrates that cell viability of the selected cell lines can be easily modulated by hydrophobic (HS) or superhydrophobic (SHS) modification of both rigid surfaces and flexible fabrics. The efficiency of coated both TCPS and PES fabrics and their effect on cell viability of the selected cell lines was evaluated using the ratio between the corresponding cell viabilities (Fig. 3B). Values higher than 1 represent conditions for which cell viability on PES fabrics are mini- mized in comparison with TCPS surfaces. Oppositely, values lower than 1 represent conditions for which cell viability on PES fabrics are preserved in comparison with TCPS surfaces.

When uncoated surfaces are considered, the obtained results suggested that cell viability became higher on flatter surfaces, as is the case of TCPS surfaces. In all cases, values lower than 1 were found. However, the corresponding values seem to be a function of the cell type. Hence, whereas this value is close to 2.5 for any of the non-tumor cell line, this ratio rise up to 16 when HeLa cell line is considered. Significant differences between all cells were found $(p<0.001)$.

On coating both TCPS and PES surfaces, the ratio values became lower, as a consequence of the reduction on cell viability. The final response, however, depends on the coated type. The superhydrophobic coating (SHS) on TCPS surfaces seems to be very efficient on decreasing cell viability, independently of the cell line type. Values lower than 1 confirmed this performance (ratios values ranged between 0.06 (3T3), 0.16 (HaCaT) and 0.43 (HeLa)). Significant differences between cell lines, uncoated surfaces and coating treatments were found.

When a more moderate coating is considered, individual responses as a function of cell line type might to be considered. Either 3T3 or HaCaT cell line decreased 10 times the ratio values in comparison with those observed on uncoated surfaces. Nevertheless, values higher than 6 demonstrated that the hydrophobic coating of PES is most efficient to minimize HeLa viability in comparison with coating TCPS surfaces. Significant differences between cells and uncoated surfaces were found $(p<0.001)$.

In this work, the influence of coating of both TCPS and PES substrates on cell response have been evaluated taking into account the substrate effect ratio (SE). SE values could be considered a parameter to express the preferential behavior on either TCPS or PES superficies. It can be seen that these values depended on both the coating treatment and the nature of the cell line. For the three cell lines, the ratio values decreased linearly as the hydrophobicity of the surface was raised. As expected, the most hydrophobic the surface, the lowest the cell viability would be. However, strong differences between selected non-tumoral and tumoral cells lines were found. The slope values vary strongly as a function of cell line with values around 1 for 3T3 and HaCaT cell lines and values higher than 8 for HeLa cell line (Table 1). The strong higher dependence of the ratio exhibited by HeLa cell line could be associated to the preference of these cells to flat surfaces, as in the case of TCPS, in comparison with $3 \mathrm{~T} 3$ and HaCaT cells.

HeLa, as well as other immortalized cell lines, are playing an increasing role in the study and development of new materials and technologies like biosensors. In fact, HeLa is the most used immortalized cell line in the laboratories all over the world and there are only few studies concerned with its adhesive properties. Lee and co-workers [51] studied the adhesion of different types of mammalian cells to modified poly(dimethylsiloxane). HeLa cells showed to be one of the most sensitive, displaying detachment and growth inhibition on several surfaces. The decrease of HeLa cell viability when compared to the control TCPS seems to be more efficient when HS modification occurs on PES fabric than in the case of flat TCPS, with ratio values close to 6.0. The significant change in substrate morphology from flat and homogeneous in TCPS to the fibrous yarned PES can play a role in discriminating cell lines according to their different ECM.

Table 1

The substrate effect (SE) was calculated as a ratio of the obtained viabilities for TCPS and PES fabrics for uncoated and coated fabrics. The slope of the fitted line is also shown.

\begin{tabular}{lllll}
\hline Cell type & $\begin{array}{l}\text { SE } \\
\text { (uncoated) }\end{array}$ & SE (HS) & SE (SHS) & Slope \\
\hline 3T3 & $2.37 \pm 0.10$ & $0.22 \pm 0.03$ & $0.06 \pm 0.007$ & 1.16 \\
HaCaT & $2.35 \pm 0.14$ & $0.17 \pm 0.01$ & $0.16 \pm 0.02$ & 1.10 \\
HeLa & $16.55 \pm 0.07$ & $6.76 \pm 0.05$ & $0.43 \pm 0.02$ & 8.06 \\
\hline
\end{tabular}


It is well established that the cell microenvironment, including the surrounding matrix, profoundly affects cell fate. This is especially true for solid tumours where matrix stiffness is believed to be an important factor in tumorogenesis [52]. It has been demonstrated that for most cell types, proliferation and cell spreading area are increased on stiffer substrates. Studies on drug screening test indicated that substrate stiffness can affect the cancer cell response to cytotoxic drugs in a cell type-dependent manner. In the case of HeLa cells, those findings suggest that stiffer substrates would be associated with higher drug resistance [53]. The significant change in substrate morphology from flat and homogeneous in TCPS to the fibrous yarned PES can play a role in discriminating cell lines, especially in the case of the tumoral HeLa cell line.

\section{Conclusions}

Depending on the coating composition and structure, the hydrophobicity of the fabric can be finely modulated by an easy-to-prepare method applicable to commercial, low cost fabric substrates providing advanced performance. This point can be regarded as a starting step to develop coated fabrics with specific wettability, chemistry and morphology. Coated-fabrics ranging from smooth and more homogeneous HS to SHS with a rougher surface in presence of nanoparticles, were obtained: In addition to the chemistry and morphology, the characteristics of the substrate are important parameter on the final cell viabilities. When uncoated surfaces are considered, the obtained results suggested that cell viability became higher on flatter surfaces, as is the case of TCPS surfaces. On coating both TCPS and PES surfaces, the ratio values became lower, as a consequence of the reduction on cell viability. The final response, however, depends on the coated type. The superhydrophobic coating (SHS) on TCPS surfaces seems to be very efficient on decreasing cell viability, independently of the cell line type. When a more moderated coating is considered, individual responses of either 3T3 or HaCaT cell line decreased 10 times the ratio values in comparison with those observed on uncoated surfaces. Nevertheless, the hydrophobic coating of PES is most efficient to minimize HeLa viability in comparison with coating TCPS surfaces. The obtained results address to a potential discrimination between tumor cell lines and non-tumor cell lines based on their adhesion on PES fabrics.

\section{Acknowledgments}

This research did not receive any specific grant from funding agencies in the public, commercial, or not-for-profit sectors. This work was initially conducted under the umbrella of COST CM1101 and MP1106 Actions. M.C. Morán and M. Ferrari acknowledge the support of UB-Mobility programme (OMPI-PR 1493) 2017-2018 call with type A1 and type A2 grants, respectively.

\section{References}

[1] D.G. Castner, B.D. Ratner, Surf. Sci. 500 (2002) 28-60.

[2] A.G. Karakecili, M. Gümüsderelioglu, Colloids Surf., B 61 (2008) 216-223.

[3] F. Chai, N. Mathis, N. Blanchemain, C. Meunier, H.F. Hildebrand, Acta Biomater. 4 (2008) 1369-1381.

[4] H. Chang, Y. Wang, in: Daniel Eberli (Ed.), Cell Responses to Surface and Architecture of Tissue Engineering Scaffolds, Regenerative Medicine and Tissue Engineering - Cells and Biomaterials, 2011, (ISBN: 978-953-307-663-8).

[5] J.M. Goddard, J.H. Hotchkiss, Prog. Polym. Sci. 32 (2007) 698-725.

[6] L.C. Xu, Biomaterials 28 (2007) 3273-3283.

[7] A.J. Engler, S. Sen, H.L. Sweeney, D.E. Discher, Cell 126 (2006) 677-689.

[8] C.B. Khatiwala, S.R. Peyton, M. Metzke, D. Putnam, J. Cell. Physiol. 211 (2007) 661-672.

[9] M. Ferrari, A. Benedetti, E. Santini, F. Ravera, L. Liggieri, E. Guzman, F. Cirisano, Colloids Surf. A Physicochem. Eng. Asp. 480 (2015) 369-375.

[10] B. Bhushan, Y.C. Jung, Prog. Mater. Sci. 56 (2011) 1-108.

[11] J.I. Lim, S. Il Kim, Y. Jung, S.H. Kim, Polymer 37 (2013) 411-419.

[12] M. Nosonovsky, B. Bhushan, J. Phys. Condens. Matter 20 (2008) 395005.
[13] E.J. Falde, S.T. Yohe, Y.L. Colson, M.W. Grinstaff, Biomaterials 104 (2016) 87-103.

[14] Y. Minghua, G. Guotuan, W.D. Meng, F.L. Qing, Appl. Surf. Sci. 253 (2007) 3669-3673.

[15] Y.I. Yoon, H.S. Moon, W.S. Lyoo, T.S. Lee, W.H. Park, Carbohydr. Polym. 75 (2009) 246-250.

[16] M. Ma, Y. Mao, M. Gupta, K.K. Gleason, G.C. Rutledge, Macromolecules 38 (2005) 9742-9748.

[17] M. Ma, R.M. Hill, J.L. Lowery, S.V. Fridrikh, G.C. Rutledge, Langmuir 21 (2005) 5549-5554.

[18] X. Zhang, F. Shi, J. Niu, Y. Jiang, Z. Wang, J. Mater. Chem. 18 (2008) 621-633.

[19] Y.Y. Yan, N. Gao, W. Barthlott, Adv. Colloid Interf. Sci. 169 (2011) 80-105.

[20] M. Ferrari, A. Benedetti, Adv. Colloid Interf. Sci. 222 (2015) 291-304.

[21] B.N. Lourenço, G. Marchioli, W. Song, R.L. Reis, C.A. van Blitterswijk, M. Karperien, A. van Apeldoorn, J.F. Mano, Biointerphases 7 (2012) 1-11.

[22] S.M. Kang, I.S. Choi, Bull. Kor. Chem. Soc. 34 (2013) 2525-2527.

[23] S. Bauer, J. Park, K. von der Mark, P. Schmuki, Acta Biomater. 4 (2008) 1576-1582.

[24] S.C. Luo, S.S. Liour, H.H. Yu, Chem. Commun. 46 (2010) 4731-4733.

[25] G.J. Toes, K.W. van Muiswinkel, W. van Oeveren, A.J.H. Suurmeijer, W. Timens, I. Stokroos, J.J.A.M. van den Dungen, Biomaterials 23 (2002) 255-262.

[26] H.Y. Wang, D.T.K. Kwok, W. Wang, Z.W. Wu, L.P. Tong, Y.M. Zhang, P.K. Chu, Biomaterials 31 (2010) 413-419.

[27] W.L. Song, D.D. Veiga, C.A. Custodio, J.F. Mano, Adv. Mater. 21 (2009) 1830-1834.

[28] N.M. Alves, J. Shi, E. Oramas, J.L. Santos, H. Tomas, J.F. Mano, J. Biomed. Mater. Res. A 91 (2009) 480-488.

[29] H.J. Busscher, I. Stokroos, J.G. Golverdingen, J.M. Schakenraad, Cell Mater. 1 (1991) 243-249.

[30] H. Schweikl, R. Muller, C. Englert, K.A. Hiller, R. Kujat, M. Nerlich, G. Schmalz, J. Mater. Sci. Mater. Med. 18 (2007) 1895-1905.

[31] J.H. Lee, G. Khang, J.W. Lee, H.B. Lee, J. Colloid Interface Sci. 205 (1998) 323-330.

[32] A. Rich, A. Harris, J. Cell Sci. 50 (1981) 1-7.

[33] H.L. Khor, Y. Kuan, H. Kukula, K. Tamada, W. Knoll, M. Moeller, D.W. Hutmacher, Biomacromolecules 8 (2007) 1530-1540.

[34] S.M. Oliveira, W. Song, N.M. Alves, J.F. Mano, Soft Matter 7 (2011) 8932-8941.

[35] C. Matschegewski, S. Staehlke, R. Loeffler, R. Lange, F. Chai, D.P. Kern, U. Beck, B.J. Nebe, Biomaterials 31 (2010) 5729-5740.

[36] M. Ferrari, F. Ravera, L. Liggieri, Appl. Phys. Lett. 88 (2006), 203125

[37] L. Liggieri, A. Passerone, High Temp. Technol. 7 (1989) 82-86.

[38] A. Sharma, J. Exp. Microbiol. Immunol. 16 (2012) 144-147.

[39] T. Mosmann, J. Immunol. Methods 65 (1983) 55-65.

[40] M.V. Berridge, P.M. Herst, A.S. Tan, Biotechnol. Annu. Rev. 11 (2005) 127-152.

[41] D. Napierska, L.C. Thomassen, V. Rabolli, D. Lison, L. Gonzalez, M. Kirsch-Volders, J.A. Martens, P.H. Hoet, Small 5 (2009) 846-853.

[42] T. Rahbari, V. Sheahan, P. Modes, C. Collier, R.M. Macfarlane, M. Badge, M. Sheahan, Collier Mc, BioTechniques 46 (2009) 277-284.

[43] V. Smith, Wonder woman: the life, death, and life after death of Henrietta lacks, unwitting heroine of modern medical science. Baltimore City Pap.

[44] N. Schurer, A. Kohne, V. Schliep, K. Barlag, G. Goerz, Exp. Dermatol. 2 (1993) 179-185.

[45] L. Micallef, F. Belaubre, A. Pinon, et al., Exp. Dermatol. 18 (2009) 143-151.

[46] G. Altankov, F. Grinnell, T. Groth, J. Biomed. Mater. Res. 30 (1996) 385-391.

[47] T. Groth, G. Altankov, J. Biomater. Sci. Polym. Ed. 7 (1995) 297-305.

[48] Y. Wang, G. Wang, X. Luo, J. Qiu, C. Tang, Burns 38 (2012) 414-420.

[49] B. Chen, W. Le, Y. Wang, Z. Li, D. Wang, L. Ren, L. Lin, S. Cui, J.J. Hu, Y. Hu, P. Yang, R.C. Ewing, D. Shi, Z. Cui, Theranostics 6 (2016) 1887-1897.

[50] C. Chatelet, O. Damour, A. Domard, Biomaterials 22 (2001) 261-268.

[51] J.N. Lee, X. Jiang, D. Ryan, G.M. Whitesides, Langmuir 20 (2004) 11684-11691.

[52] D.E. Discher, P. Janmey, Y. Wang, Science 310 (2005) 1139-1143.

[53] S. Zustiak, R. Nossal, D.L. Sackett, Biotechnol. Bioeng. 111 (2014) 396-403.

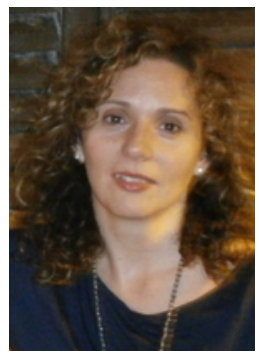

M. Carmen Morán (BS-Chemistry-1996; MS-Chemistry-1997; PhD-Chemistry-2002 UB) is Associate Professor at the Department of Biochemistry and Physiology, Faculty of Pharmacy and Food Science at the University of Barcelona (UB). She obtained her $\mathrm{PhD}$ investigating the enzymatic synthesis of biocompatible amino acid-based surfactants at the Department of Surfactant Technology-Spanish Council for Scientific Research (IIQAB-CSIC). By means of UE founding (Marie Curie-Research Training Network CIPSNAC and NEONUCLEI, among other) she involved in the preparation and physicochemical characterization of DNA gel particles during her postdoctoral stage at the Department of Chemistry at the University of Coimbra-Portugal (2006-2010). Through a Ramon y Cajal contract, she incorporated at the Faculty of Pharmacy and Food Science (2010-). Her scientific background is now being applied to more biologically-oriented questions as the preparation of efficient therapeutic delivery systems and their in vitro biocompatibility characterization. M. C. Morán authored $>70$ works referenced in peer reviewed journal, one hundred communications in scientific meeting and seven patents. 
Barcelona. During this time, he involved in the preparation of drug delivery systems and their cytotoxicity and potential selectivity towards selected non-tumoral and tumoral cell lines under bulk and in textiles substrates conditions. He is currently a PhD student in the Department of Chemical Engineering at the Polytechnic University of Catalonia. His research is focused on conducting polymers as platforms for biomedical applications.

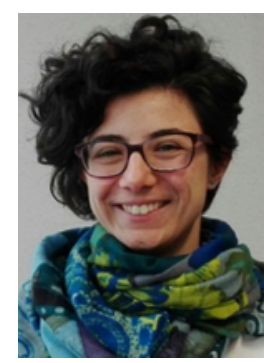

Francesca Cirisano received her M.Sc. in Chemical Sciences in 2013 from the University of Genoa and subsequently, after a fellowship at the CNR-ICMATE (National Research Council-Institute of Condensed Matter Chemistry and Technologies for Energy), she obtained her $\mathrm{PhD}$ in Sciences and Technologies of Chemistry and Materials in 2017 from University of Genoa/CNR-ICMATE. Actually, she is a postdoctoral research fellow at the CNR-ICMATE. Her research interest includes the fabrication of coatings with special wettability, self-cleaning and antifouling properties for marine and biomedical application.

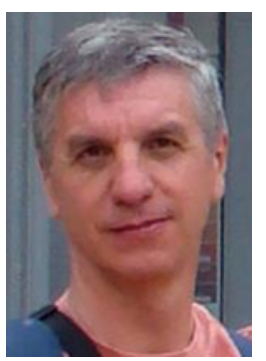

Michele Ferrari is Staff Researcher at CNR-ICMATE (Institute of Condensed Matter and Technologies for Energy) in Genova, Italy. After the studies of Chemistry in the field of protection in cultural heritage (University of Genoa), he joined as a postdoc the group of Dr. Miller in 1994 at Max Planck Inst. for Colloids and Interfaces in Berlin. He has been visiting researcher (2001) at MPI-KG (Golm-Germany), MPI-PR (Mainz-Germany) (2006), UNISA (Adelaide-Australia) (2009) and INFLPR (Magurele-Romania) (2013). Member of COST European Action networks in Physics of Droplets (P21), Colloidal Aspects of Nanoscience for Innovative Processes and Materials (CM1101) and Smart and green interfaces (MP1106) - from single bubbles and drops to industrial, environmental and biomedical applications. His scientific interests include wetting, superhydrophobic coatings, application in marine environment, interfacial properties of surfactant systems and AFM and 3D-prolifometry in studies of adsorption and self-assembly of amphiphiles at liquid-solid interface. Co-author of $>90$ articles in peer reviewed journals, books, encyclopedia, and co-editor of books and special issues and $>100$ contributions at international conferences. 